\title{
Single-Projection Based Volumetric Image Reconstruction and 3D Tumor Localization in Real Time for Lung Cancer Radiotherapy
}

\author{
Ruijiang Li, Xun Jia, John H. Lewis, Xuejun Gu, Michael Folkerts, \\ Chunhua Men, and Steve B. Jiang \\ Department of Radiation Oncology, University of California San Diego \\ 3855 Health Sciences Dr. 0843, La Jolla, CA 92037, USA \\ \{rul002, xunjia, jlewis, x2gu, mfolkert, cmen, sbjiang\}@ucsd.edu
}

\begin{abstract}
We have developed an algorithm for real-time volumetric image reconstruction and 3D tumor localization based on a single x-ray projection image. We first parameterize the deformation vector fields (DVF) of lung motion by principal component analysis (PCA). Then we optimize the DVF applied to a reference image by adapting the PCA coefficients such that the simulated projection of the reconstructed image matches the measured projection. The algorithm was tested on a digital phantom as well as patient data. The average relative image reconstruction error and 3D tumor localization error for the phantom is $7.5 \%$ and $0.9 \mathrm{~mm}$, respectively. The tumor localization error for patient is $\sim 2$ $\mathrm{mm}$. The computation time of reconstructing one volumetric image from each projection is around 0.2 and 0.3 seconds for phantom and patient, respectively, on an NVIDIA C1060 GPU. Clinical application can potentially lead to accurate $3 \mathrm{D}$ tumor tracking from a single imager.
\end{abstract}

Keywords: image reconstruction, tumor localization, lung motion, GPU, lung cancer radiotherapy.

\section{Introduction}

Management of tumor motion is a challenging and important problem for modern highly conformal lung cancer radiotherapy. Poorly managed tumor motion can lead to poor target coverage and an unnecessarily high dose to normal tissues [1]. Therefore, precise knowledge of real-time lung tumor motion during the treatment delivery is essential for the effectiveness of lung cancer radiotherapy [2-8]. In [9-11] 3D respiratory motion was estimated from cone beam projections based on a generic B-spline motion model. However, because of the large number of parameters in the model, many projections over a large range of angles have to be used. Therefore, the estimation process is retrospective and cannot be done in real time.

The goal of this work is to demonstrate the feasibility of extracting lung motion information from a single $\mathrm{x}$-ray projection in real time. This is achieved with effective use of the prior information provided by 4DCT or 4DCBCT, where the deformation of the entire lung at different phases is efficiently represented by principal component 
analysis (PCA). The inherent regularization imposed by the PCA lung motion model allows us to obtain the volumetric image of the patient from a single projection. Realtime efficiency is achieved by implementing the algorithm on a graphics processing unit (GPU).

We organize the paper as follows. In section 2, we briefly introduce the PCA lung motion model and furnish the reason behind its suitability for our goal. Then we use the PCA model to reconstruct volumetric images and localize tumors with an appropriate cost function. In section 3, we provide some details on the phantom and patient data which the algorithm was tested on. In section 4 , we demonstrate the results. We conclude the paper in section 5 .

\section{Methods}

The basic idea of our work is to first obtain a lung motion model parameterized by a few PCA coefficients, and then adapt the deformation vector field (DVF) applied to a reference $\mathrm{CT}$ such that its projection matches the x-ray projection images acquired during the treatment. In the following, whenever we mention CT or 4DCT, the same principle can be applied to CBCT or 4DCBCT too.

\subsection{PCA Lung Motion Model}

Our method starts by parameterizing lung motion with PCA. The PCA lung motion model was first proposed by [12] and was recently shown in [13] to bear a close relationship with the physiological 5D lung motion model [14] on a theoretical basis. In the PCA model, lung motion (i.e., motion of each voxel in the lung along each of the 3 spatial coordinates) denoted as a vector function $\mathbf{x}(t)$, can be approximated by a linear combination of the eigenvectors corresponding to the largest eigenvalues:

$$
\mathbf{x}(t) \approx \overline{\mathbf{x}}+\sum_{k=1}^{K} \mathbf{u}_{k} w_{k}(t)
$$

where $\mathbf{u}_{k}$ are the eigenvectors obtained from PCA and are functions of space only. The scalars $w_{k}(t)$ are PCA coefficients and are functions of time only. It is worth mentioning that the eigenvectors are fixed after PCA and it is the evolution of the PCA coefficients that drives the dynamic lung motion.

There are primarily two reasons why the PCA lung motion model is suitable for this work. First, PCA provides the best linear representation of the data in the least mean-square-error sense. Second, the PCA motion model imposes inherent regularization on its representation. One can show that $\|\Delta \mathbf{u}\|^{2} \leq \sum_{i}\left(1 / \sigma_{i}^{2}\right) \cdot\|\Delta \mathbf{x}\|^{2}$, where $\sigma_{i}$ are the eigenvalues from PCA. This means that if two voxels move similarly, their motion represented by PCA will also be similar [13]. The combined effect is that a few scalar variables (PCA coefficients) are sufficient to dynamically deform the lung in a reasonably accurate way. 
In order to build a PCA lung motion model, a representative lung motion state for one breathing cycle needs to be available for training purposes. In practice, this can be obtained from deformable image registration (DIR) between a reference CT phase and all other phases in a 4DCT (or 4DCBCT) data set, which is available from the treatment simulation (or setup).

\subsection{Image Reconstruction Using the PCA Model}

After we have obtained a parameterized PCA lung motion model, we seek a set of optimal PCA coefficients such that the simulated projection of the reconstructed CT matches well with the measured x-ray projection. But the simulated and measured projection may have very different intensity levels. Here we assume there exists a linear relationship between them. The cost function is:

$$
\begin{aligned}
& \min . J(\mathbf{w}, a, b)=\left\|\mathbf{P} \cdot \mathbf{f}\left(\mathbf{x}, \mathbf{f}_{0}\right)-a \cdot \mathbf{y}-b \cdot \mathbf{1}\right\|_{2}^{2} \\
& \text { s.t. } \quad \mathbf{x}=\overline{\mathbf{x}}+\mathbf{U} \cdot \mathbf{w}
\end{aligned}
$$

where, $\mathbf{U}$ and $\mathbf{w}$ are comprised of a set of eigenvectors and PCA coefficients, $\mathbf{x}$ is the parameterized DVF, $\mathbf{f}_{0}$ is the reference CT, $\mathbf{f}$ is the reconstructed CT, $\mathbf{y}$ is the projection image, and $\mathbf{P}$ is a projection matrix which calculates the simulated projection. For simplicity, we have suppressed the time index under $\mathbf{w}, \mathbf{x}$, and $\mathbf{y}$.

To find the optimal values for $\mathbf{w}, a, b$, the algorithm alternates between the following 2 steps:

$$
\begin{aligned}
& \text { step 1: } \mathbf{w}_{n+1}=\mathbf{w}_{n}-\mu_{n} \cdot \frac{\partial J}{\partial \mathbf{w}_{n}} \\
& \text { step 2: }\left(a_{n+1}, b_{n+1}\right)^{T}=\left(\mathbf{Y}^{T} \mathbf{Y}\right)^{-1} \mathbf{Y}^{T} \mathbf{P} \mathbf{f}_{n+1} \\
& \text { where, } \mathbf{Y}=[\mathbf{y}, \mathbf{1}] \text {, and } \frac{\partial J}{\partial \mathbf{w}}=\frac{\partial \mathbf{x}}{\partial \mathbf{w}} \cdot \frac{\partial \mathbf{f}}{\partial \mathbf{x}} \cdot \frac{\partial J}{\partial \mathbf{f}}=\mathbf{U}^{T} \cdot \frac{\partial \mathbf{f}}{\partial \mathbf{x}} \cdot \mathbf{P}^{T} \cdot(\mathbf{P} \cdot \mathbf{f}-a \cdot \mathbf{y}-b \cdot \mathbf{1})
\end{aligned}
$$

Given the new DVF at each iteration, the reconstructed CT $\mathbf{f}$ is found through trilinear interpolation. Accordingly, $\partial \mathbf{f} / \partial \mathbf{x}$ has to be consistent with the interpolation process in order to get the correct gradient. It turns out that $\partial \mathbf{f} / \partial \mathbf{x}$ is a linear combination of the spatial gradients of the image evaluated at the neighboring 8 grid points, weighted by the appropriate fractional part of the DVF. The step size $\mu_{n}$ in step 1 is found by Armijo's rule for line search. In step 2, the update for $a, b$ is the unique minimizer of the cost function with fixed $\mathbf{w}$. Therefore, the cost function always decreases at each step. Note that the cost function is lower bounded by zero. The above alternating algorithm is guaranteed to converge for all practical purposes. The algorithm stops whenever the norm of the gradient is sufficiently small or the maximum number of iterations (10 in this paper) is reached. 


\subsection{Tumor Localization by Deformation Inversion}

In order to get the correct tumor position, we distinguish between 2 different kinds of DVFs: push forward DVF and pull back DVF. The DVF found by (2) is a pull back DVF. It cannot be used directly to calculate the new tumor position. To do that, we need its inverse, i.e., the push forward DVF. Here, we adopt a fixed-point approach for deformation inversion [15] and calculate tumor position.

\section{Materials}

\subsection{Digital Phantom}

The algorithm was tested using a non-uniform rational B-spline (NURBS) based cardiac-torso (NCAT) phantom [16]. This mathematical phantom has a high level of anatomical realism (e.g., a beating heart, detailed bronchial trees). The respiratory motion was developed based on basic knowledge of respiratory mechanics. We generated a dynamic NCAT phantom composed of 10 phases as our simulated 4DCT. The NCAT phantom also outputs the $3 \mathrm{D}$ tumor position, which is used as ground truth. The CT data dimension is: $256 \times 256 \times 120$ (voxel size: $2 \times 2 \times 2.5 \mathrm{~mm}^{3}$ ).

We used the end of exhale (EOE) phase as the reference image and did DIR between EOE phase and all other phases. The DIR algorithm is a fast demons algorithm implemented on GPU [17]. Then PCA was performed on the 9 DVFs from DIR and 3 PCA coefficients and eigenvectors were kept in the PCA model. We simulated x-ray images at different phases from those for training with different breathing amplitudes as well as at different gantry angles using Siddon's algorithm [18]. The imager dimension is down-sampled to $200 \times 150$ (pixel size: $2 \times 2 \mathrm{~mm}^{2}$ ).

\subsection{Patient Data}

The algorithm was also evaluated with a patient data set. 4DCT of the patient was acquired using a GE four-slice LightSpeed CT scanner (GE Medical Systems, Milwaukee, WI, USA) and the RPM system. The cone beam projections were taken with the Varian on-board imaging system (Varian Medical Systems, Palo Alto, CA, USA) in half-fan mode with $110 \mathrm{kVp}, 20 \mathrm{~mA}$ and $20 \mathrm{~ms}$ exposure time. For the patient in this study, there were no implanted fiducial makers and real-time 3D location of the tumor was not available to evaluate our algorithm. Instead, we projected the estimated $3 \mathrm{D}$ tumor location onto the $2 \mathrm{D}$ imager and compared with that manually defined by a clinician. From the clinician-defined contour, the tumor centroid position was calculated for each projection and used as the ground truth to evaluate the algorithm. We calculated the tumor localization error along the axial and tangential directions, both scaled back to the mean tumor position.

\section{Results}

\subsection{Phantom Results}

We tested the extrapolation performance of our algorithm by generating a new CT volume where there is an increase of 50\% in breathing amplitude (tumor motion 

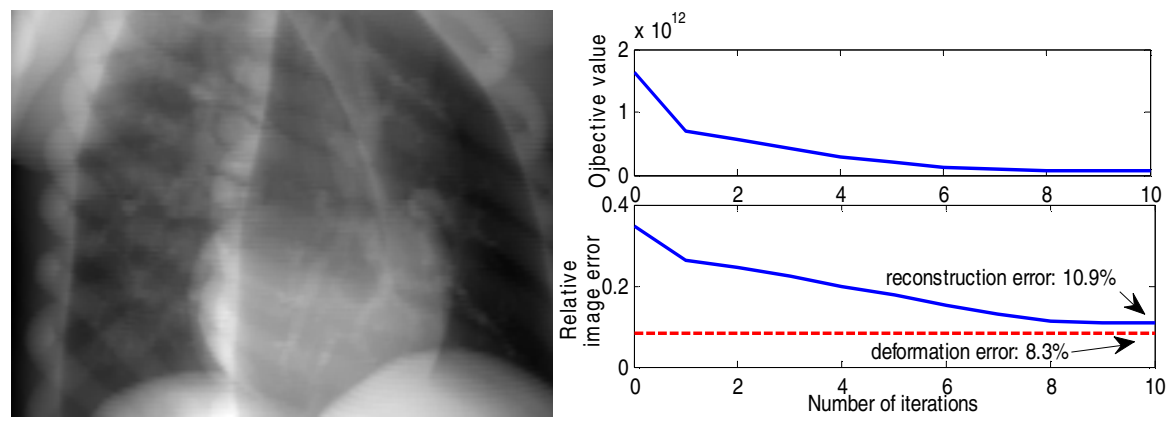

Fig. 1. a): "measured" projection of the test image at a right posterior oblique (RPO) angle; b): objective value (top) and the relative image reconstruction error (bottom) at each iteration

a)

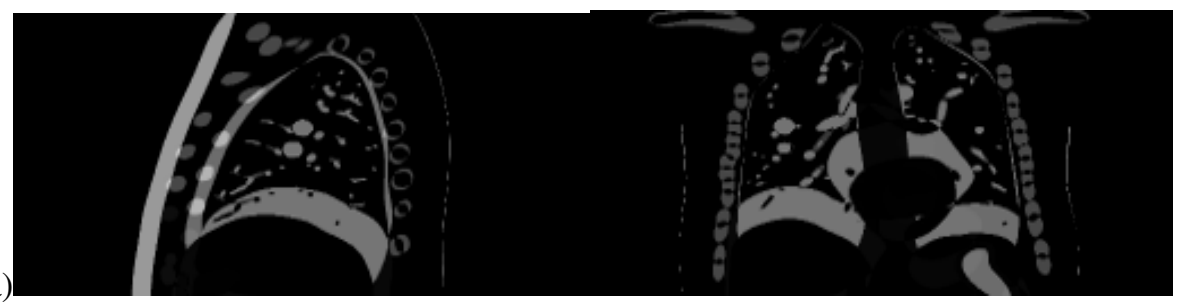

b)

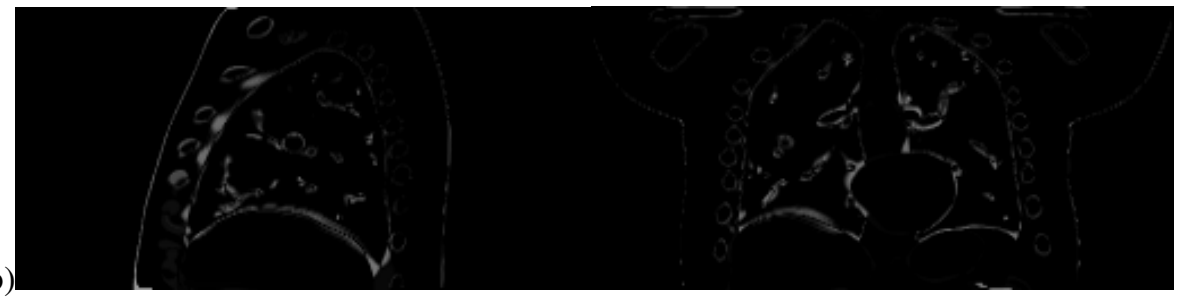

c)

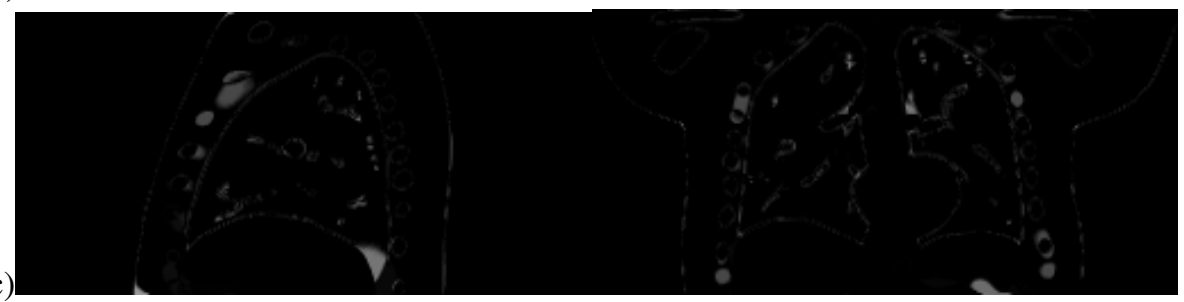

Fig. 2. Left column: sagittal view of the absolute difference image between a), test and reference $\mathrm{CT}$; b), test $\mathrm{CT}$ and $\mathrm{CT}$ reconstructed using proposed algorithm; c), test CT and deformed CT using demons. Tumor is a round object near the center of the slice. Right column: same as left column, except for coronal view. Tumor is a round object in the right lung.

magnitude: $16 \mathrm{~mm}$ in training versus $24 \mathrm{~mm}$ in testing). The simulated projection image (see Fig. 1) was generated from the end of inhale phase so that it is maximally different from the reference CT. The algorithm converges within 10 iterations. Figure 2 shows the sagittal and coronal views of the reference CT and the difference images. The relative 3D RMS image reconstruction error is initially $35 \%$ and approaches to that obtained by 3D DIR with demons (11\% compared with $8 \%$ ). Note 
that demons uses the 3D test CT instead of 2D projection image used by our algorithm. The 3D RMS tumor localization error is $0.9 \mathrm{~mm}$.

We then did a more systematic evaluation by generating a dynamic phantom consisting of 60 phases with a 50\% increase in breathing amplitude and 4-sec period. We simulated cone beam $x$-ray projections at all angles with spacing of $1^{\circ}$ resulting in 360 projections in 1 minute (15 breathing cycles). The average relative 3D image reconstruction error is $7.5 \% \pm 2.4 \%$. The average $3 \mathrm{D}$ tumor localization error is 0.9 $\mathrm{mm} \pm 0.5 \mathrm{~mm}$ and is not affected by projection angles (see Fig. 3).

In order to speed up the computation, we have implemented our algorithm on an NVIDIA C1060 GPU. We initialized the PCA coefficients as those from previous frame in order to get further reduction in computation time. The image reconstruction and tumor localization for each projection was achieved between 0.2 and 0.3 seconds.



Fig. 3. Top row: relative image error between the ground truth test image under regular breathing and: reference image (dashed line); image reconstructed using the proposed algorithm (solid line) as a function of cone beam projection angle. Bottom row: same as top row, except for 3D localization error.

\subsection{Patient Results}

For the patient, approximately 650 projections were acquired over a full gantry rotation with a frequency of about $10.7 \mathrm{~Hz}$. However, since the cone beam scans were performed in the half-fan mode (with the imager shifted about $14.8 \mathrm{~cm}$ laterally), and isocenter of the scan was not placed at the tumor, the tumor is only visible in a subset of these projections. The tumor was marked by the clinician in the largest continuous set of projections in which the tumor was visible. For this patient, 281 projections were used.
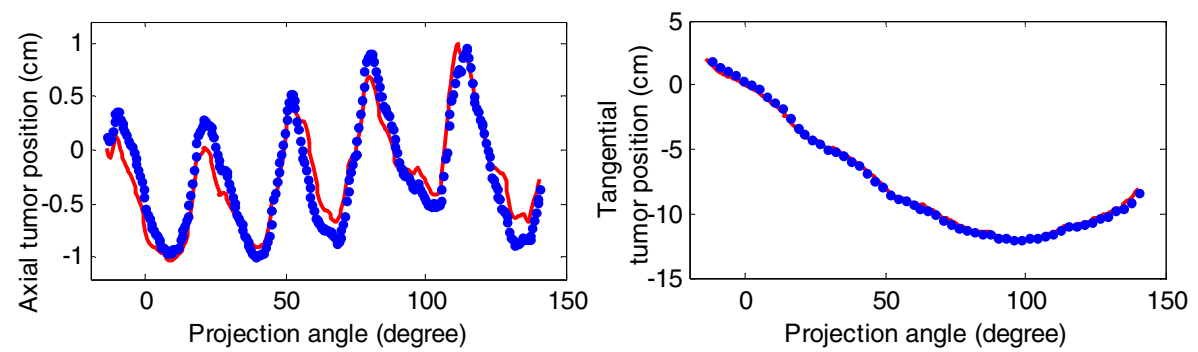

Fig. 4. Tumor localization results for the patient. Dots represent the algorithm output, and the solid lines represent the clinician marked tumor position. 
The patient had quite irregular breathing during the CBCT scan, with both baseline drift and amplitude changes. Figure 4 shows the tumor localization results. For the axial direction the average localization error is $1.9 \pm 0.9 \mathrm{~mm}$. In the tangential direction the average error is $1.8 \pm 1.0 \mathrm{~mm}$. The average computation time for image reconstruction and tumor localization from each projection was around 0.3 seconds on an NVIDIA Tesla C1060 GPU card.

\section{Conclusions}

We have shown it is feasible to extract lung motion information from a single $\mathrm{x}$-ray projection within half a second. To further speed up the computation, we can predict the current PCA coefficients using previous histories and use that as the starting point. We plan to comprehensively evaluate the accuracy of the algorithm on more clinical data. It is worth mentioning that careful quality assurance (e.g., patient breathing coaching during 4DCT acquisition), better DIR algorithms will help improve the algorithm's accuracy on patient data. Also, it is not clear if the PCA motion model would be able to capture dramatic changes in breathing patterns between treatment simulation and different treatment fractions. Should that occur, 4D CBCT acquired on the day of treatment may be more appropriate to build the PCA motion model.

\section{References}

1. Keall, P.J., Mageras, G.S., Balter, J.M., Emery, R.S., Forster, K.M., Jiang, S.B., Kapatoes, J.M., Low, D.A., Murphy, M.J., Murray, B.R., Ramsey, C.R., Van Herk, M.B., Vedam, S.S., Wong, J.W., Yorke, E.: The management of respiratory motion in radiation oncology report of AAPM Task Group 76. Med. Phys. 33, 3874-3900 (2006)

2. Cervino, L.I., Chao, A.K., Sandhu, A., Jiang, S.B.: The diaphragm as an anamic surrogate for lung tumor motion. Phys Med. Biol. 54, 3529-3541 (2009)

3. Berbeco, R.I., Nishioka, S., Shirato, H., Jiang, S.B.: Residual motion of lung tumors in end-of-inhale respiratory gated radiotherapy based on external surrogates. Med. Phys. 33, 4149-4156 (2006)

4. Hoisak, J.D., Sixel, K.E., Tirona, R., Cheung, P.C., Pignol, J.P.: Correlation of lung tumor motion with external surrogate indicators of respiration. Int. J. Radiat. Oncol. Biol. Phys. 60, 1298-1306 (2004)

5. Wu, H., Zhao, Q., Berbeco, R.I., Nishioka, S., Shirato, H., Jiang, S.B.: Gating based on internal/external signals with dynamic correlation updates. Phys. Med. Biol. 53, 7137-7150 (2008)

6. Cui, Y., Dy, J.G., Sharp, G.C., Alexander, B., Jiang, S.B.: Multiple template-based fluoroscopic tracking of lung tumor mass without implanted fiducial markers. Phys. Med. Biol. 52, 6229-6242 (2007)

7. Xu, Q., Hamilton, R.J., Schowengerdt, R.A., Alexander, B., Jiang, S.B.: Lung Tumor Tracking in Fluoroscopic Video Based on Optical Flow. Medical Physics 35, 5351-5359 (2008)

8. Lin, T., Cervino, L.I., Tang, X., Vasconcelos, N., Jiang, S.B.: Fluoroscopic tumor tracking for image-guided lung cancer radiotherapy. Phys. Med. Biol. 54, 981-992 (2009)

9. Zeng, R., Fessler, J.A., Balter, J.M.: Estimating 3-D respiratory motion from orbiting views by tomographic image registration. IEEE Trans. Med. Imaging 26, 153-163 (2007) 
10. Vandemeulebroucke, J., Kybic, J., Clarysse, P., Sarrut, D.: Respiratory Motion Estimation from Cone-Beam Projections using a Prior Model. In: Medical Image Computing and Computer-Assisted Intervention, vol. 5762, pp. 365-372. Springer, London (2009)

11. Fluck, O., Aharon, S., Khamene, A.: Efficient Framework for Deformable 2D-3D Registration. Medical Imaging: Visualization, Image-guided Procedures, and Modeling (2008)

12. Zhang, Q., Pevsner, A., Hertanto, A., Hu, Y.C., Rosenzweig, K.E., Ling, C.C., Mageras, G.S.: A patient-specific respiratory model of anatomical motion for radiation treatment planning. Med. Phys. 34, 4772-4781 (2007)

13. Li, R., Lewis, J.H., Jia, X., Zhao, T., Lamb, J., Yang, D., Low, D.A., Jiang, S.B.: PCAbased lung motion model. In: 16th International Conference on the Use of Computers in Radiation Therapy, Amsterdam, Netherlands (2010) (accepted)

14. Low, D.A., Parikh, P.J., Lu, W., Dempsey, J.F., Wahab, S.H., Hubenschmidt, J.P., Nystrom, M.M., Handoko, M., Bradley, J.D.: Novel breathing motion model for radiotherapy. Int. J. Radiat. Oncol. Biol. Phys. 63, 921-929 (2005)

15. Chen, M., Lu, W., Chen, Q., Ruchala, K.J., Olivera, G.H.: A simple fixed-point approach to invert a deformation field. Med. Phys. 35, 81-88 (2008)

16. Segars, W.P., Lalush, D.S., Tsui, B.M.W.: Modeling respiratory mechanics in the MCAT and spline-based MCAT phantoms. IEEE Transactions on Nuclear Science 48, 89-97 (2001)

17. Gu, X., Pan, H., Liang, Y., Castillo, R., Yang, D., Choi, D., Castillo, E., Majumdar, A., Guerrero, T., Jiang, S.B.: Implementation and evaluation of various demons deformable image registration algorithms on a GPU. Phys. Med. Biol. 55, 207-219 (2010)

18. Siddon, R.L.: Fast calculation of the exact radiological path for a three-dimensional CT array. Medical Physics 12, 252-255 (1985) 